\title{
Chemodosimeter-based fluorescent detection of L-cysteine after extracted by molecularly imprinted polymers
}

\author{
Xiaoqiang Cai ${ }^{\mathrm{a}, \mathrm{b}, \mathrm{c}, 1}$, Jinhua Li ${ }^{\mathrm{b}, \mathrm{c}, 1}$, Zhong Zhang ${ }^{\mathrm{b}, \mathrm{c}, \mathrm{e}}$, Gang Wang ${ }^{\mathrm{b}, \mathrm{c}}$, Xingliang Song ${ }^{\mathrm{d}}$, \\ Jinmao You ${ }^{\mathrm{a}}$, Lingxin Chen ${ }^{\mathrm{a}, \mathrm{b}, \mathrm{c}, *, *}$ \\ a College of Chemistry and Chemical Engineering, Qufu Normal University, Qufu 273165, China \\ ${ }^{\mathrm{b}}$ Key Laboratory of Coastal Environmental Processes and Ecological Remediation, Yantai Institute of Coastal Zone Research, Chinese Academy of Sciences, \\ Yantai 264003, China \\ ' Shandong Provincial Key Laboratory of Coastal Environmental Processes, Yantai Institute of Coastal Zone Research, Chinese Academy of Sciences, \\ Yantai 264003, China \\ d School of Chemistry \&' Chemical Engineering, Linyi University, Linyi 276005, China \\ e University of Chinese Academy of Sciences, Beijing 100049, China
}

\section{A R T I C L E I N F O}

\section{Article history:}

Received 18 October 2013

Received in revised form

4 December 2013

Accepted 5 December 2013

Available online 15 December 2013

Keywords:

Molecularly imprinted polymer

Fluorescence detection

L-cysteine

Chemodosimeter

Rhodamine B

\begin{abstract}
A B S T R A C T
A chemodosimeter-based fluorescent detection method coupled with molecularly imprinted polymers (MIPs) extraction was developed for determination of L-cysteine (L-Cys) by combining molecular imprinting technique with fluorescent chemodosimeter. The MIPs prepared by precipitation polymerization with L-Cys as template, possessed high specific surface area of $145 \mathrm{~m}^{2} / \mathrm{g}$ and good thermal stability without decomposition lower than $300{ }^{\circ} \mathrm{C}$, and were successfully applied as an adsorbent with excellent selectivity for L-Cys over other amino acids, and enantioselectivity was also demonstrated. A novel chemodosimeter, rhodamine B1, was synthesized for discriminating L-Cys from its structurally similar homocysteine and glutathione as well as various possibly co-existing biospecies in aqueous solutions with notable fluorescence enhancement when adding L-Cys. As L-Cys was added with increasing concentrations, an emission band peaked at $580 \mathrm{~nm}$ occurred and significantly increased in fluorescence intensity, by which the L-Cys could be sensed optically. High detectability up to $12.5 \mathrm{nM}$ was obtained. An excellent linearity was found within the wide range of $0.05-50 \mu \mathrm{M}(r=0.9996)$, and reasonable relative standard deviations ranging from $0.3 \%$ to $3.5 \%$ were attained. Such typical features as high selectivity, high sensitivity, easy operation and low cost enabled this MIPs-fluorometry to be potentially applicable for routine detection of trace L-Cys.
\end{abstract}

(c) 2013 Elsevier B.V. All rights reserved.

\section{Introduction}

L-cysteine (L-Cys), a sulfur containing amino acid, is of great significance in biological systems owing to its crucial roles in a variety of fundamental physiological processes [1,2]. Many significant cellular functions are aided by L-Cys such as detoxification and metabolism. Both deficiency and elevated levels of L-Cys are involved in organism abnormality, such as liver damage, hematopoiesis decrease and neurotoxicity [1-3]. Therefore, identifying and quantifying L-Cys is very important and considerable efforts have been made to develop high efficient methods for its determination [4-6]. Common separation techniques such as high performance liquid chromatography (HPLC), capillary electrophoresis (CE) and gas chromatography (GC), coupled with fluorescence or electrochemical

\footnotetext{
* Corresponding author: Yantai Institute of Coastal Zone Research, Chinese Academy of Sciences, Yantai 264003, China. Tel./fax: +86535 2109130.

E-mail address: lxchen@yic.ac.cn (L. Chen).

${ }^{1}$ Equally contributed to this work.
}

detection, are usually utilized for assaying L-Cys, but they are often inconvenient to operate and often require time consuming sample preparation and separation procedures [7,8]. Because L-Cys possesses a very low molar absorptivity and lacks a suitable chromophore, derivatization with fluorescent reagents is typically used for detection [9]. Most of these fluorescence-labeling reagents contain functional groups which can react with thiol groups in general, and hence are often not specific to L-Cys [10]. Recent research have focused on the development of direct fluorescent methods for the detection of LCys and other biological thiols, aiming at high sensitivity, remote operation, and suitability as a fluorescent tag for labeling [11,12]. Among them, the fluorescence turn-on mode, either increase in fluorescence intensity or a new photoluminescence at a different wavelength, has been increasingly developed for mercapto biomolecules [13-15]. For example, Hao et al. [13] demonstrated a fluorescence turn-on molecular probe for direct and highly selective detection of Cys using a zwitterionic dye. Li et al. [15] devised and synthesized a chemodosimeter (also named chemosensor $[16,17]$ ) which could effectively discriminate Cys from homocysteine and 
glutathione with notable fluorescence enhancement. Inspired by these studies, we proposed to synthesize a new fluorescent chemodosimeter possessing excellent selectivity for L-Cys.

On the other hand, it is imperative to highly efficiently extract and separate trace L-Cys in diverse complex matrices. For this purpose, a promising material, molecularly imprinted polymers (MIPs) can be employed based on its selective recognition and high adsorption capabilities [18]. The target analytes will be separated from coexisting substances and selectively adsorbed on the MIPs materials, followed by detection [19]. Because of its attractive selectivity, high mechanical and thermal stability, high adsorption capacity, as well as low cost and easy operation, MIPs have received extensive concerns and been widely applied in many fields, such as extraction separation $[20,21]$, removal [22] and chemo/biosensors [23,24]. A number of studies for amino acids based on MIPs have been rapidly carried out. Especially, electrically conductive MIPs and enantioselective recognition are recently emerged research hotspots [25-27], such as electrosynthesized imprinted polypyrrole films for enantioselective recognition of L-aspartic acid [25], and colorimetric sensing of amino acids chiral recognition based on molecularly imprinted photonic polymers [27]. However, as far as we are aware, no MIPs-based extraction followed by fluorescent detection for L-Cys has been reported.

In this work, we conceive to recognize and extract L-Cys by using MIPs as adsorbent, and then perform fluorescence determination by using a newly synthesized chemodosimeter of rhodamine B derivative, named RB1 for simplicity. The MIPs exhibited high specific surface area, good thermal stability, and high adsorption capacity. The new RB1 displayed an excellent selectivity for L-Cys over structure-similar homocysteine and glutathione. The combination of MIPs and RB1 enabled that excellent analytical merits were obtained by the newly developed fluorescence spectrophotometric method for L-Cys analysis.

\section{Experimental}

\subsection{Materials and instruments}

L-cysteine (L-Cys), homocysteine (Hcy) and glutathione (GSH) were purchased from Aladdin (Shanghai, China). Methacrylic acid (MAA), ethyleneglycol dimethacrylate (EGDMA), rhodamine B (RB) and hydrazine hydrate were purchased from Sigma-Aldrich (Shanghai, China) and distilled under vacuum prior to use to remove stabilizers. 2,2'-Azo-bis-isobutyronitrile (AIBN) was obtained from Sinopharm Chemical Reagent Co. Ltd. (Shanghai, China) and recrystallized in methanol prior to use. D-cysteine (D-Cys) was purchased from Sigma-Aldrich (Shanghai, China). Threonine (Thr), glucose (Gluc), glycine (Gly), tryptophan (Trp), lysine (Lys), leucine (Leu), tyrosine (Tyr), histidine (His), taurine (Tau), serine (Ser), phenylalanine (Phe), glutamine (Gln), asparagine (Asn), proline (Pro), alanine (Ala), arginine (Arg), methionine (Met), and aspartic acid (Asp) were provided by Shanghai Shenggong Co. (Shanghai, China). Chromatographic grade acetonitrile $(\mathrm{ACN})$ and methanol $(\mathrm{MeOH})$ were attained from J\&K Chemical (Beijing, China). Other affiliated reagents were all provided by Sinopharm Chemical Reagent Co. Ltd. (Shanghai, China). All solvents and chemicals were of analytical grade and used without further purification unless otherwise specified. Deionized water used throughout the work was produced by a Milli-Q Ultrapure water system with the water outlet operating at 18.2 $\mathrm{M} \Omega$ (Millipore, Bedford, MA, USA).

Fluorescence spectra were conducted on a HORIBA Fluoromax-4 spectrofluometer (France). SEM images were recorded using a scanning electron microscope (SEM, Hitachi S-4800, Japan) operating at an accelerating voltage of $5 \mathrm{kV}$. All samples were sputtercoated with gold before SEM analysis. $\mathrm{N}_{2}$ adsorption-desorption isotherms and structure parameters (specific surface area, pore size and volume) were obtained by Full-automatic Specific Surface Instruments (3H-2000BET-A, Beishide Instruments, Beijing, China). Thermogravimetric (TG) analysis about thermostability and purity was performed by a ZRY-2P thermal analyzer (Mettler Toledo).

\subsection{Preparation of $L$-Cys MIPS}

The L-Cys MIPs were prepared by precipitation polymerization based on non-covalent approach, by using MAA as functional monomer, EGDMA as cross-linker and the mixture of ACN and $\mathrm{MeOH}$ as porogen. The preparation process is schematically depicted in Scheme 1. Briefly, prior to polymerization, pre-polymer solutions were prepared by dissolving MAA ( $4 \mathrm{mmol})$ and $\mathrm{L}-\mathrm{Cys}(1 \mathrm{mmol})$ in the mixture of $\mathrm{ACN}(30 \mathrm{~mL})$ and $\mathrm{MeOH}(10 \mathrm{~mL})$, which were stored at $4{ }^{\circ} \mathrm{C}$ in dark for $12 \mathrm{~h}$. Then, EGDMA (20 mmol) and AIBN (20 mg) were added. The solution was degassed in an ultrasonic bath for $5 \mathrm{~min}$, and then was purged with nitrogen for $10 \mathrm{~min}$ while cooling in an ice bath for $10 \mathrm{~min}$. Then, a two-step polymerization was carried out in a water bath at $50{ }^{\circ} \mathrm{C}$ for $6 \mathrm{~h}$, followed by $24 \mathrm{~h}$ at $60^{\circ} \mathrm{C}$. The resultant polymer particles were separated and washed with methanol/acetic acid solution $(9: 1, v / v)$ to remove both the template molecules and residual monomers. Finally, the particles were dried to constant weight under vacuum at $40^{\circ} \mathrm{C}$. For comparison, nonimprinted polymers (NIPs) were prepared under identical conditions but without the template in the reaction system.

\subsection{Static adsorption test}

The static adsorption test was carried out by allowing a certain amount of MIPs to reach the equilibrium (the adsorption equilibrium would be obtained within $20 \mathrm{~h}$ ) with L-Cys standard solution of various known concentrations. Briefly, the MIPs of $20 \mathrm{mg}$ were dispersed in a $5 \mathrm{~mL}$ flask containing $2.0 \mathrm{~mL}$ L-Cys solutions at different concentrations $(10,20,30,40$ and $50 \mu \mathrm{M})$, followed by nitrogen input to purge oxygen. The mixture was shaken (180 times/min) for $24 \mathrm{~h}$ in ice-bath at room temperature with a horizontal shaker and then separated centrifugally (4000 rpm) for $5 \mathrm{~min}$. The supernatant was measured for the unbound L-Cys by fluorescence spectrometry at the excitation wavelength of $525 \mathrm{~nm}$. The amounts of L-Cys adsorbed onto the MIPs were calculated by subtracting the amounts of unbound compounds from the amounts of compounds added to the mixture. The same procedure was applied to test the static adsorption of NIPs.

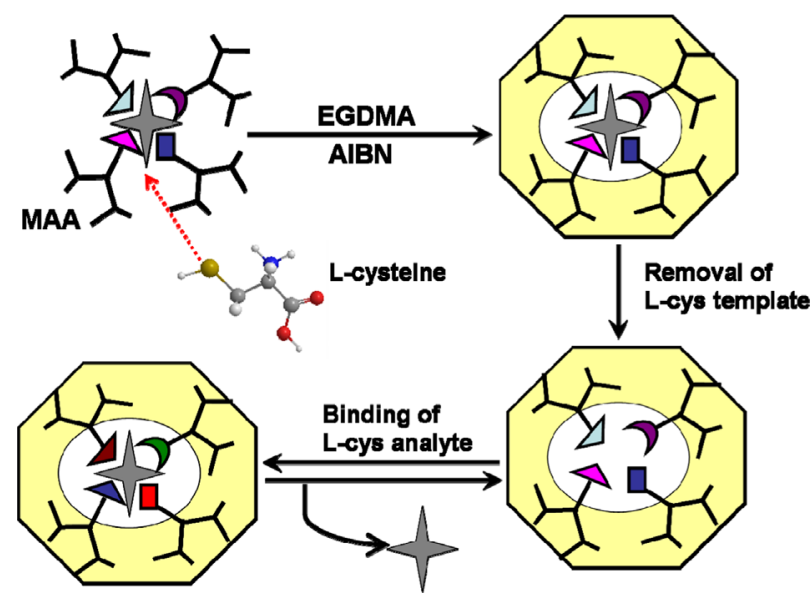

Scheme 1. Schematic representation of MIPs preparation for L-Cys. 


\subsection{Selectivity of MIPs}

Selectivity experiments were conducted by using enantiomer D-Cys, structurally similar Hcy and GSH, and other amino acids such as Gly, Arg and His. HPLC-UV with precolumn phenylisothiocyanate (PITC) derivatization was employed for determination as follows. For derivatization, $100 \mu \mathrm{L}$ of PITC at $0.01 \mathrm{M}$ dissolved in acetonitrile and $100 \mu \mathrm{L}$ of triethylamine at $1 \mathrm{M}$ in acetonitrile were added to $200 \mu \mathrm{L}$ of amino acid solutions, mixed, and were set aside for $1 \mathrm{~h}$ at room temperature. And then $400 \mu \mathrm{L}$ of $n$-hexane was added, keeping an oscillation for 2 min by using a swirling commingler, and then the mixture was centrifuged for $10 \mathrm{~min}$ at $8000 \mathrm{rpm}$, followed by a placement for $10 \mathrm{~min}$. The lower liquid was pipetted, and was filtrated with a $0.45 \mu \mathrm{m}$ filter, for HPLC analysis. An HPLC instrument (Agilent HP 1100 series) with diode array detector (DAD) was used. UV-vis detection wavelength was set at $254 \mathrm{~nm}$. HPLC separation was performed by injecting $10 \mu \mathrm{L}$ samples onto a $\mathrm{C}_{18}$ column (Symmetry Shield $\mathrm{RP}_{8}, 5 \mu \mathrm{m}$, $200 \mathrm{~mm} \times 4.6 \mathrm{~mm}$ i.d., Waters, USA), and column temperature was set at $38^{\circ} \mathrm{C}$. A mobile phase system consisted of two components, namely solvent $A(0.1 \mathrm{M}$ sodium acetate/acetonitrile $(97: 3, \mathrm{v} / \mathrm{v}))$ and solvent $\mathrm{B}$ (acetonitrile $/ \mathrm{H}_{2} \mathrm{O}(80: 20, \mathrm{v} / \mathrm{v})$ ). A gradient program was started from $10 \% \mathrm{~B}$ and held for $10 \mathrm{~min}$, then increased linearly to $75 \%$ B within $10 \mathrm{~min}$, and then increased to $100 \%$ B within $5 \mathrm{~min}$, and held for another $5 \mathrm{~min}$ before re-conditioning, at a flow rate of $1.0 \mathrm{~mL} / \mathrm{min}$.

\subsection{Synthesis of RB1}

RB1 was synthesized based on RB by a two-step reaction according to previously reported method [22] with necessary modification, and the synthetic route was briefly illustrated in Scheme 2. Briefly, $1.2 \mathrm{~g}(2.5 \mathrm{mmol})$ of $\mathrm{RB}$ and $30 \mathrm{~mL}$ of ethanol were put in a $100 \mathrm{~mL}$ flask. During vigorously agitating at room temperature, $3.0 \mathrm{~mL}$ of $85 \%$ hydrazine hydrate was added dropwise, followed by a refluxing for $2 \mathrm{~h}$. After that, the mixed solution changed to dark-violet from light-brown, and finally to transparent. The solution was cooled down, and then ethanol was distillated at reduced pressure. And then $50 \mathrm{~mL}$ of $\mathrm{HCl}(1 \mathrm{M})$ was added, and a red solution was obtained. Under continuously stirring, $70 \mathrm{~mL}$ of $\mathrm{NaOH}(1 \mathrm{M})$ was added until pH reaching 9-10, and a lot of white precipitates showed up. They were filtrated and washed triplicates using $15 \mathrm{~mL}$ of water. After dried under vacuum, the product, named N3 for simplicity, was obtained. Then $478.0 \mathrm{mg}$ of $\mathrm{N} 3(1.0 \mathrm{mmol})$ was added into $100 \mathrm{~mL}$ flask, followed by $30 \mathrm{~mL}$ absolute ethanol and $2.0 \mathrm{~mL} 40 \%$ glyoxal (excess), and then the mixture was stirred for $2 \mathrm{~h}$ under nitrogen protection at room temperature. After solvent distillated at reduced pressure, silica gel (200-300 mesh) separation was carried out by using the eluent of petroleum ether and ethyl acetate $(4: 1, \mathrm{v} / \mathrm{v})$. The resultant yellow solid was characterized and confirmed by ${ }^{1} \mathrm{H}$ NMR and ${ }^{13} \mathrm{C}$ NMR spectroscopy (Fig. S1), and mass spectrometry (Fig. S2). For simplicity, it was named RB1.

\section{Results and discussion}

\subsection{Characterization of MIPS}

Fig. 1 shows the SEM images of L-Cys MIPs and corresponding NIPs prepared by precipitation polymerization. As displayed, although both have rough surfaces, the MIPs seemed more dense and homogenous with more and larger-dimension pores in the network (Fig. 1a) than NIPs (Fig. 1b). The roughness of the MIPs surface should be considered as a factor providing an increase in the surface area that would reduce diffusion resistance and facilitate mass transfer. The mean size of the MIP microparticles was estimated to be $255 \mathrm{~nm}$ measured by ZetaCAD (Fig. S3). More importantly, the uniform and more open structure is obviously favorable for the embedding of the template molecules and mass transfer. Moreover, their nitrogen adsorption-desorption results were obtained, i.e., the specific surface area, pore volume and pore size of MIPs were $145 \mathrm{~m}^{2} / \mathrm{g}, 0.56 \mathrm{~cm}^{3} / \mathrm{g}$ and $15.58 \mathrm{~nm}$, respectively, while $120 \mathrm{~m}^{2} / \mathrm{g}, 0.42 \mathrm{~cm}^{3} / \mathrm{g}$ and $14.05 \mathrm{~nm}$ of NIPs. These results indicated that the imprinted structure significantly increased the specific surface area by using L-Cys to create specific polymeric micropores, a large number of L-Cys could enter into the MIPs and predominantly occupy the pores and cavities, and thereby would accelerate mass transfer and improve adsorption capacity, which is a desirable property of an efficient adsorbent as a bed of SPE and/ or HPLC columns [28].

Fig. 2a shows the TG curves of MIPs and NIPs. With the temperature increasing from 50 to $100{ }^{\circ} \mathrm{C}$, their weight losses were mainly due to the loss of absorbed water. In the range of $100-290{ }^{\circ} \mathrm{C}$, the rate of weight loss for MIPs was very slow, whereas a high rate of weight loss occurred at temperatures ranging from 290 to $450{ }^{\circ} \mathrm{C}$. The peak temperature of MIPs was $382.38^{\circ} \mathrm{C}$ and the residue amount was $48.23 \%$. The weight loss might result from the decomposing of polymers. However, notably, the TG curve of NIPs began to descend over $200{ }^{\circ} \mathrm{C}$ and DTG curve showed two change trends (Fig. 2). Therefore, the prepared L-Cys-MIPs were fully demonstrated to have excellent thermal stability at lower than $300^{\circ} \mathrm{C}$. These can also be clearly explained from Fig. 2 b.

\subsection{Static adsorption estimation of the MIPS}

Fig. 3a shows the binding isotherms of L-Cys onto the MIPs and NIPs. The curves were obtained by plotting the saturated adsorption amounts with equilibrium concentrations of the L-Cys in the mixed solution of ACN and $\mathrm{MeOH}$. As seen, the adsorption capacity of L-Cys increased quickly with the increase of initial concentration. At equilibrium concentrations higher than $50.0 \mu \mathrm{M}$, adsorption of MIPs became stable and its recognition sites were almost saturated. NIPs had the same trend as MIPs but with much lower saturated adsorption amounts, because a large number of binding sites were formed in the MIPs and they were specific to L-Cys. Thus, static adsorption isotherms are important for describing how adsorbates interact with adsorbent [29].

Scatchard analysis was employed to further evaluate the binding isotherms. According to the Scatchard equation [29], the plot

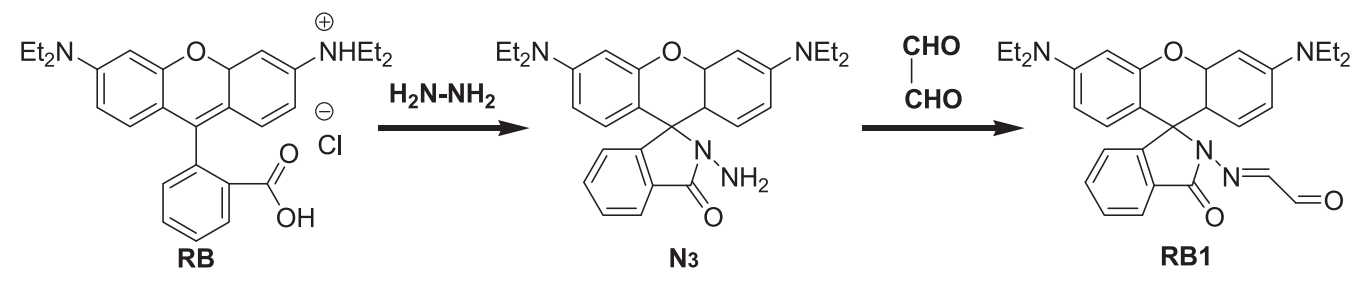

Scheme 2. Synthetic route of RB1. 

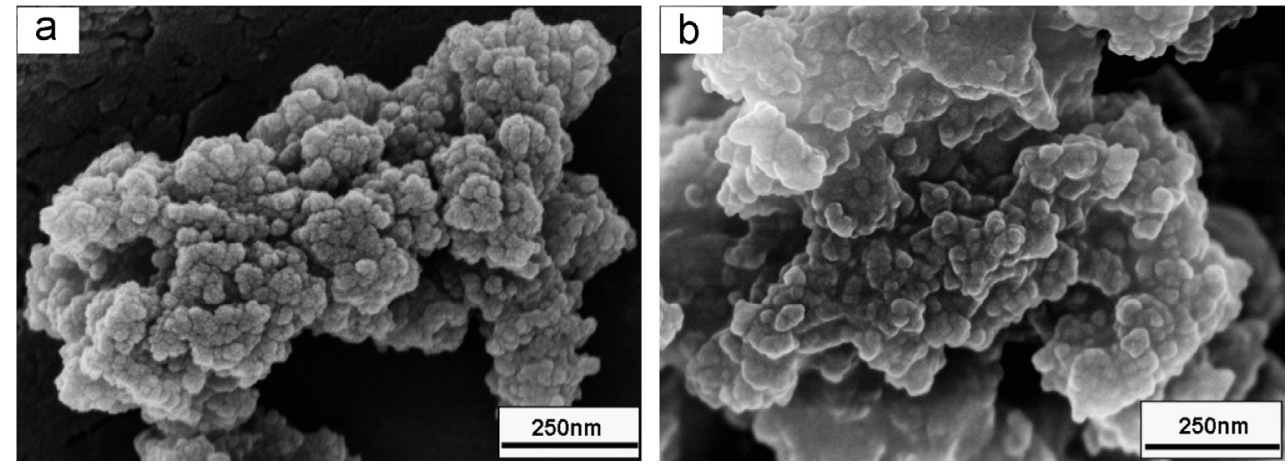

Fig. 1. SEM images of MIPs (a) and NIPs (b).

a

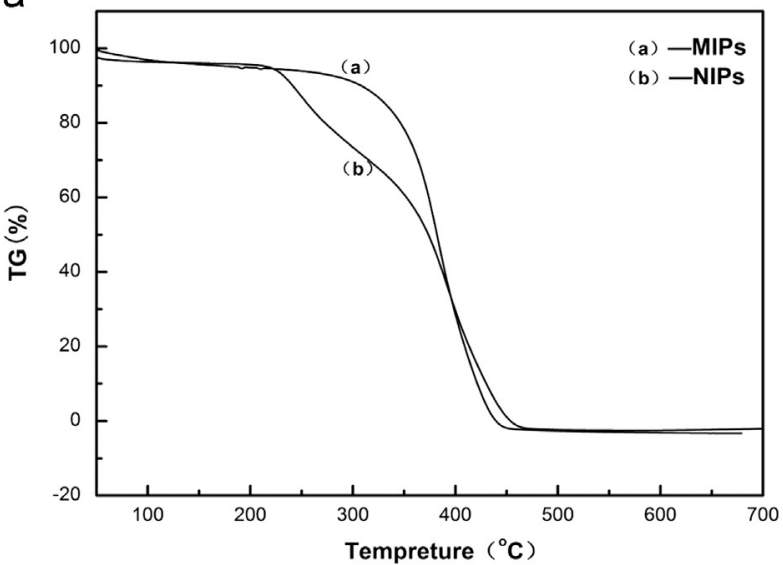

b

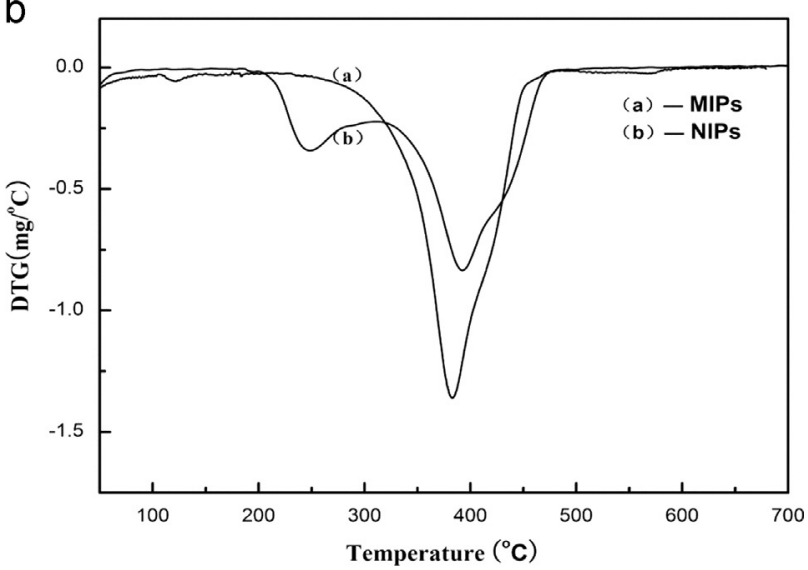

Fig. 2. (a) TG and (b) DTG analysis curves of MIPs and NIPs.

for L-Cys adsorbed onto MIPs was obtained. And consequently, the $K_{\mathrm{d}}$ (equilibrium dissociation constant) and $Q_{\max }$ (maximum adsorption capacity) were calculated, i.e., $13.04 \mathrm{mg} / \mathrm{L}$ and 1.790 $\mathrm{mg} / \mathrm{g}$ for MIPs, and $9.020 \mathrm{mg} / \mathrm{L}$ and $0.5874 \mathrm{mg} / \mathrm{g}$ for NIPs, respectively, and thereby the imprinting factor was attained of 3.0. The results further confirmed that MIPs possessed unique template molecule adsorption properties. As for the sorption isotherm models, Langmuir and Freundlich isotherm parameters for adsorption of L-Cys onto the MIPs are displayed in Table 1. Their corresponding equations are given in Appendix A. It can be observed that the Langmuir isotherm model yielded a better fit than that by the Freundlich model, with the correlation coefficients $\left(R^{2}\right)$ above 0.999 . As seen, Fig. 3b shows the equilibrium
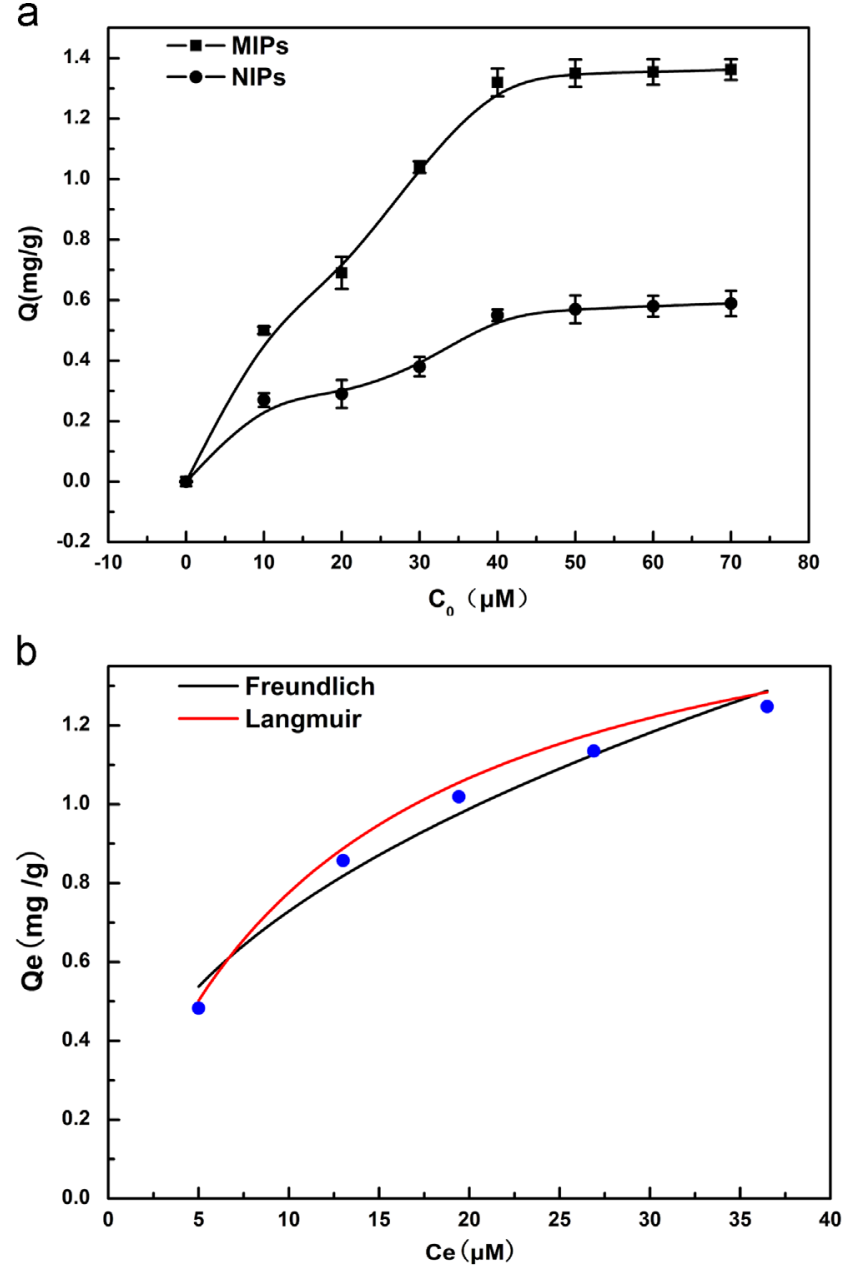

Fig. 3. (a) Binding isotherms of MIPs and NIPs for L-Cys and (b) fitting using Langmuir isotherm model for L-Cys adsorption onto MIPs. $Q$, adsorption capacity; $Q_{e}$, equilibrium adsorption capacity; $C_{0}$, initial concentration of L-Cys; $C_{e}$, equilibrium concentration of L-Cys. Experimental conditions: $\mathrm{V}=2 \mathrm{~mL}$; mass of polymer $20 \mathrm{mg}$; adsorption time, $24 \mathrm{~h}$.

data for Langmuir and Freundlich isotherms, further confirming that the Langmuir isotherm model is suitable to the MIPs adsorption for L-Cys.

\subsection{Binding selectivity of MIPS}

In order to further investigate the competitive recognition and adsorption ability of the MIPs, one enantiomer (D-Cys), two 
Table 1

Langmuir and Freundlich isotherm model parameters for the MIPs and NIPs.

\begin{tabular}{llcc}
\hline Isotherm model & Parameters & MIPs & NIPs \\
\hline Langmuir & $R^{2 \mathrm{a}}$ & 0.9993 & 0.9980 \\
& $Q_{\max }^{\mathrm{b}}$ & 1.644 & 0.5690 \\
& $K_{L}^{\mathrm{c}}$ & 0.0832 & 0.110 \\
Freundlich & $R^{2}$ & 0.9722 & 0.9873 \\
& $K_{\mathrm{F}}{ }^{\mathrm{d}}$ & 19.4355 & 21.3580 \\
& $1 / n^{\mathrm{e}}$ & 0.5365 & 0.7752
\end{tabular}

\footnotetext{
a Correlation coefficient.

${ }^{\mathrm{b}}$ Maximum binding capacity.

${ }^{\mathrm{c}}$ Langmuir constant.

${ }^{\mathrm{d}}$ Indicative constant for adsorption capacity of the adsorbent.

e Ranging from 0 to 1 , measuring the adsorption intensity or surface hetero-
} geneity.

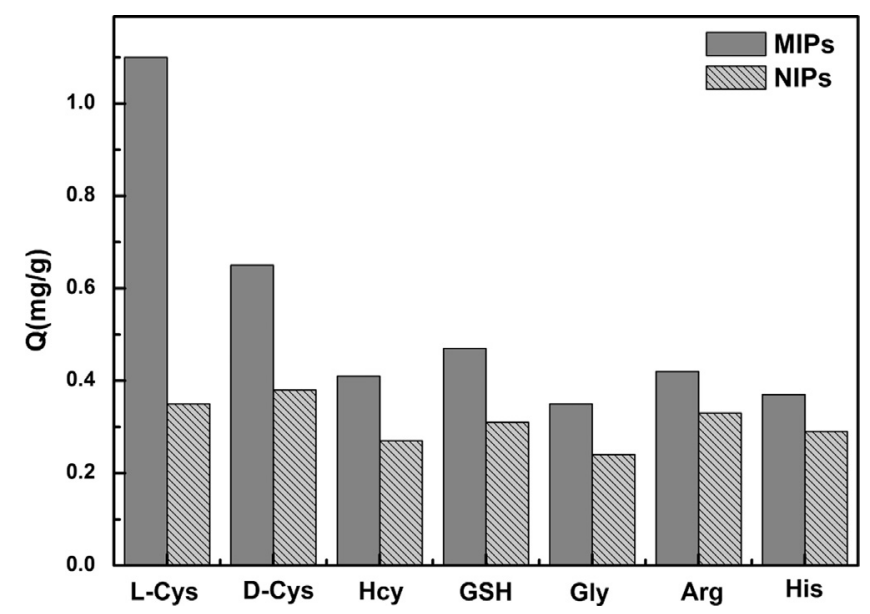

Fig. 4. Binding capacities of MIPs and NIPs for L-Cys, D-Cys, Hcy, GSH, Gly, Arg and His. Experimental conditions: $\mathrm{V}=2 \mathrm{~mL}$; mass of polymer, $20 \mathrm{mg}$; concentration of amino acid, $30 \mu \mathrm{M}$; adsorption time, $24 \mathrm{~h}$.

mercapto amino acids (Hcy and GSH) and three typical amino acids (Gly, Arg and His) were chosen as references. As seen from Fig. 4, for the MIPs, the binding capacities for L-Cys were the highest, and D-Cys showed higher binding capacities than the other five amino acids as its structure is more similar to L-Cys. It is seen that the adsorption capacities of NIPs were very close for all the tested amino acids, because there were not selective recognition sites in the NIPs and the adsorption for subjects was non-selective. On the other hand, obviously, the MIPs offered significantly better selectivity for L-Cys since the MIPs presented the capacity value about 3 times of that for the corresponding NIPs. The molecular recognition of MIPs lies on that the generated binding sites should be complementary to the template, in size, shape and orientation of their functional groups [25]. So the obtained L-Cys MIPs could selectively recognize and bind L-Cys from D-Cys and other amino acids. The functionalized MIPs opened a promising way as an attractive sorbent to selective preconcentration of L-Cys.

\subsection{Fluorescent detection of L-Cys via RB1}

Based on the above results, the L-Cys could be highly effectively enriched and extracted from aqueous solutions by the prepared MIPs, and then fluorescent detection was followed. It is well known the free RB1 is weakly fluorescent and colorless. The maximum UV-vis absorption band is at $556 \mathrm{~nm}$, and $510-540 \mathrm{~nm}$ is usually employed as the excitation wavelength in order to avoid self-quenching.
So, herein, we chose $525 \mathrm{~nm}$ as the excitation wavelength. When L-Cys was added with increasing concentrations, an emission band peaked at $580 \mathrm{~nm}$ appeared and significantly increased in fluorescence intensity, as shown in Fig. 5. The fluorescence intensity of RB1 solution exhibited an excellent linearity to the concentration of L-Cys in the range of $0.05-50 \mu \mathrm{M}(r=0.9996)$ with relative standard deviations between $0.3 \%$ and $3.5 \%$ (inset of Fig. 5), indicating that L-Cys could be accurately detected in a wide quantitation range with high reliability. Based on $3 \delta_{\text {blank }} / k$ (where $\delta_{\text {blank }}$ is the standard deviation of the blank solution and $k$ is the slope of the calibration plot), the limit of detection (LOD) was attained of $12.5 \mathrm{nM}$. A comparison of the analytical performances with that previously reported methods $[4-6,8,11,13,15,30-35]$ for the determination of L-Cys is summarized in Table 2. As can be seen from the table, the present method is an ideal candidate for L-Cys analysis. The improvement of the detection sensitivity could be attributed to the high enrichment ability of MIPs for L-Cys. Besides the imprinting effect, RB1 played a crucial role in sensitivity enhancement as a new fluorescence-enhanced L-Cys chemodosimeter. The results indicated that RB1 could assist to sensitively detect biological relevant levels of L-Cys after extraction and preconcentration by MIPs.

The possible detection mechanism for the optical changes was investigated. The free RB1 is colorless and weakly fluorescent. As illustrated in Scheme 3, upon addition of L-Cys to the RB1 solution, an unstable intermediate of $\mathbf{1}$ (thiazolidine derivative) was formed, followed by a ring-opening reaction that could promote the hydrolysis of $\mathbf{2}-\mathbf{3}$. And the reaction would induce strong fluorescence accompanied by color change from colorless to pink, since a strong conjugate system was formed in $\mathbf{3}$ (Scheme 3 ). This postulation can be supported by that reported in references $[15,36]$. Therefore, the fluorescent detection strategy could be established by using the new chemodosimeter of RB1. This recognition for $\mathrm{L}-\mathrm{Cys}$ should be attributed to the special reactions of RB1 with L-Cys via the formation and hydrolysis of a thiazolidine derivative.

\subsection{Molecular selectivity and sensing specificity}

The selective reaction of RB1 was evaluated by using L-Cys and its structurally similar compounds, Hcy and GSH, chosen as

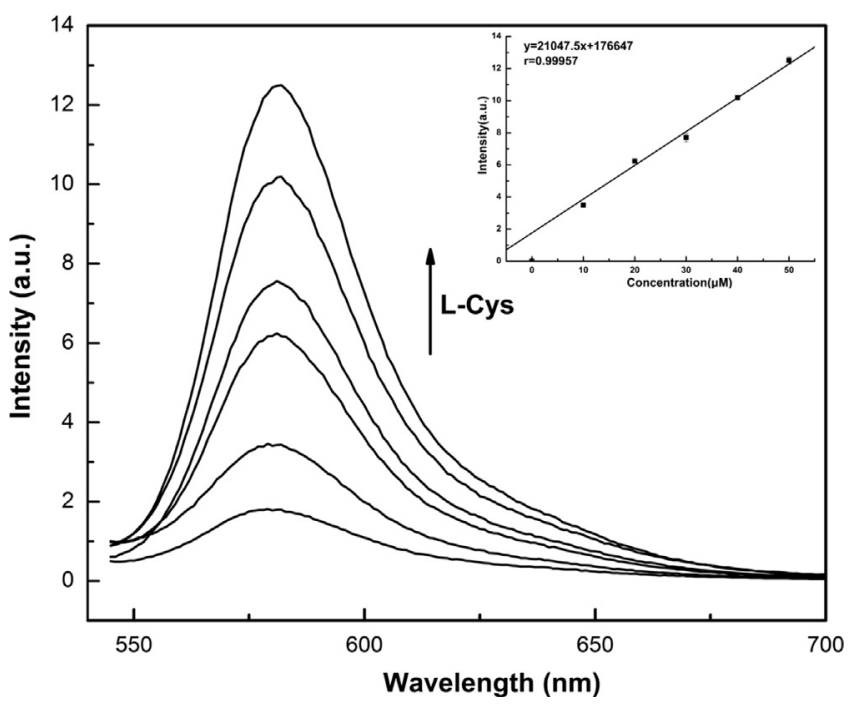

Fig. 5. Fluorescence spectra of $\mathrm{RB} 1(10 \mu \mathrm{M})$ solution upon addition of L-Cys (0$50 \mu \mathrm{M})$. Each spectrum was recorded after $30 \mathrm{~min}$. Inset: plot of the fluorescence intensity as a function of the concentration of L-Cys. 
Table 2

Comparison of method performances for determination of L-Cys.

\begin{tabular}{|c|c|c|c|c|}
\hline Detection method & Technique in detail & Linear range $(\mu \mathrm{M})$ & LOD (nM) & Ref \\
\hline Fluorimetry & MIPs preconcentration combing with a newly synthesized chemodosimeter of RB1 & $0.05-50$ & 12.5 & This work \\
\hline Fluorimetry & Surface-modified CdS NPs as a fluorescent probe & $0.5-10$ & - & [11] \\
\hline Fluorimetry & Colorimetric and near-infrared fluorescence turn-on molecular probe using a zwitterionic dye of M1 & - & $4.07(\mu \mathrm{M})$ & [13] \\
\hline Fluorimetry & A synthesized chemodosimeter of RS1 & - & 73.5 & [15] \\
\hline Fluorimetry & Redox reaction between the $\mathrm{Cu}^{2+}$-morin complex and Cys & $0.6-22$ & 65.2 & [30] \\
\hline Electrochemistry & Metal-organic framework for electrocatalytic oxidation & $0.02-300$ & 8 & [6] \\
\hline Electrochemistry & Carbon paste electrode modified with $\mathrm{Co}(\mathrm{II})-\mathrm{Y}$ zeolite & $1 \mathrm{nM}-1 \mathrm{mM}$ & 0.237 & [31] \\
\hline Electrochemistry & AuNPs/poly-eriochrome black T film modified glassy carbon electrode & $0.05-100$ & 8 & [32] \\
\hline Electrochemistry & Reversion of fluorescence quenching of calcein by copper(II) ion & $0.3-12$ & 40 & [4] \\
\hline $\mathrm{SPR}^{\mathrm{a}}$ & DNA intercalator-based amplification & $0.05-2$ & 50 & {$[5]$} \\
\hline Colorimetry & AuNPs stabilized with ssDNA ${ }^{\mathrm{b}}$ & $0.1-1.3$ & 100 & [33] \\
\hline Colorimetry & Carboxymethyl cellulose-functionalized AuNPs & $10.0-100.0$ & - & [34] \\
\hline Chromatography & Monolithic RPLC-MS ${ }^{\mathrm{C}}$ & $0.25-50$ & 36 & [35] \\
\hline Chromatography & Transient pseudo-isotachophoretic stacking in $\mathrm{CZE}^{\mathrm{d}}$ & $5-80$ & $1(\mu \mathrm{M})$ & [8] \\
\hline
\end{tabular}

a Surface plasmon resonance.

b Single-stranded oligonucleotide.

${ }^{c}$ Reversed-phase liquid chromatography-mass spectrometry.

${ }^{\mathrm{d}}$ Capillary zone electrophoresis.
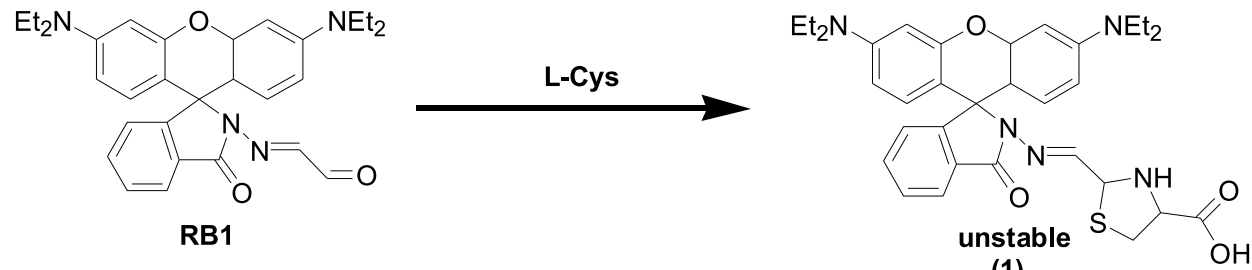

(1)

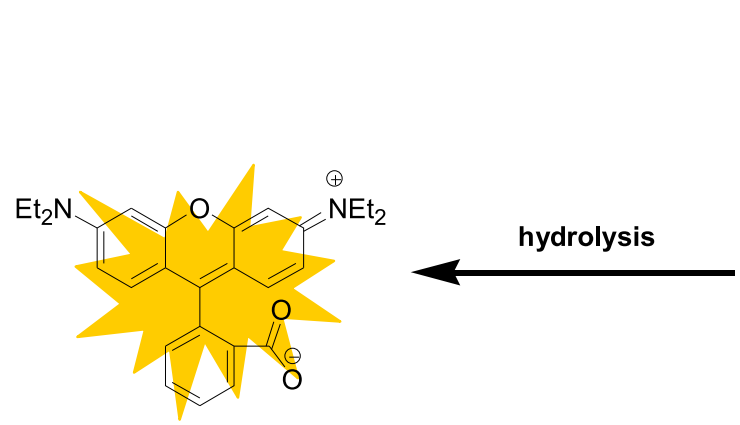

(3)

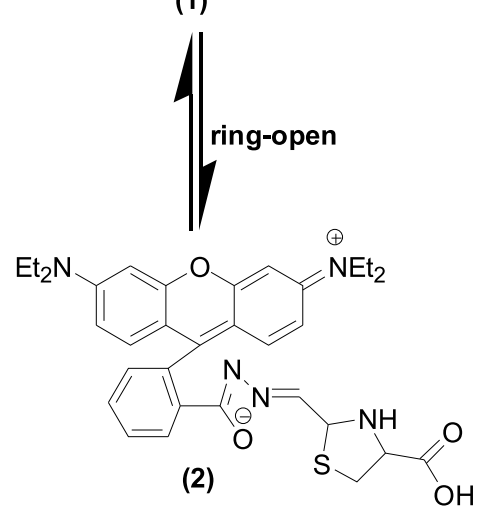

Scheme 3. Proposed mechanism of fluorescent detection for L-Cys.

references for investigating the optical properties. When adding Hcy and GSH to the RB1 solution, no obvious fluorescence change was observed. For Hcy, a stable complex was produced, but it was colorless and non-fluorescent. In the case of GSH, the SH group could not react with RB1 since there is no adjacent $\mathrm{NH}_{2}$ group. As seen in Fig. 6a, the time dependent fluorescence intensity changes of RB1 solution showed much larger for L-Cys than Hcy and GSH, which might well be from the generally more favored 5-membered ring heterocycles, as compared to 6-membered ring formation of Hcy, upon reaction with the unsaturated aldehyde [15]. Excitedly, upon addition of L-Cys, followed by a corresponding spectrum recorded every $5 \mathrm{~min}$, the fluorescence intensity gradually increased with the time increasing, and then reached stable after $60 \mathrm{~min}$. And when $40 \mu \mathrm{M}$ L-Cys was added, about 7-fold enhancement in intensity was attained. Meanwhile, the competition and interference experiments were also carried out. As shown in Fig. 6b, various possibly present species did not significantly influence the fluorescence enhancement of RB1 solutions by L-Cys, except that Hcy induced a small decrease of fluorescence intensity. The results clearly demonstrated that RB1 was an excellent L-Cys-specific fluorescent chemodosimeter.

\section{Conclusions}

In summary, we presented a new method of MIPs extraction coupled with fluorescent quantitation based on chemodosimeter for L-Cys determination. The newly prepared MIPs proved to be an efficient selective adsorbent for enrichment of trace level L-Cys, and the newly synthesized RB1 was demonstrated a specific chemodosimeter for fluorescent detection, which successfully discriminated L-Cys from Hcy, GSH and other amino acids in aqueous solutions. Taking advantage of the high adsorption capability of MIPs and the super fluorescence-enhancement property of RB1, the developed system achieved excellent analytical performances toward L-Cys, such as high sensitivity $(12.5 \mathrm{nM})$ and wide linear range $(0.05-50 \mu \mathrm{M})$. Further explorations are expected in order to improve the detection sensitivity and to extend the method to other mercapto biomolecules. With the delicate design and synthesis of new chemodosimeter, the amino acid/protein determination optical sensing platform can be constructed for selective, sensitive and rapid routine monitoring of organism status. Moreover, the combination of MIPs and chemodosimeter 

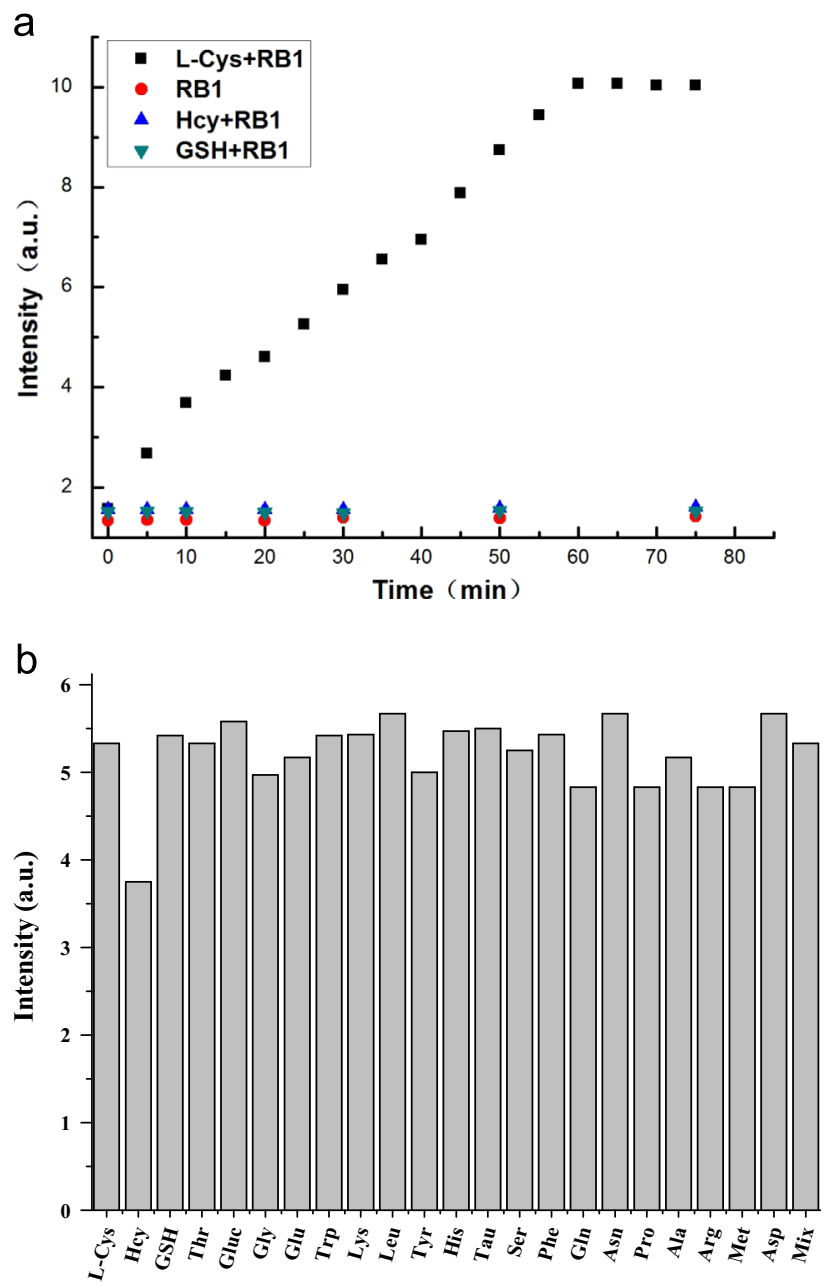

Fig. 6. (a) Time dependent fluorescence intensity changes of RB1 $(10 \mu \mathrm{M})$ in the absence and presence of 20 eq. Cys, Hcy and GSH. (b) Fluorescence responses of RB1 $(10 \mu \mathrm{M})$ solution at $552 \mathrm{~nm}$ to L-Cys and to the mixture of L-Cys with other bio-species; $\quad \mathrm{Mix}=\mathrm{Hcy}+\mathrm{GSH}+\mathrm{Thr}+\mathrm{Gluc}+\mathrm{Gly}+\mathrm{Trp}+\mathrm{Lys}+\mathrm{Leu}+\mathrm{Try}+\mathrm{His}+\mathrm{Tau}+$ Ser + Phe + Gln + Asn + Pro + Ala + Arg + Met + Asp, $200 \mu$ M respectively.

will greatly enrich the research contents as well as broaden the applications of molecular imprinting techniques.

\section{Acknowledgments}

This work was financially supported by the National Natural Science Foundation of China (21105117, 21275158, 21275068, 2127
5089), the Natural Science Foundation of Shandong Province of China (ZR2010BQ027), and the 100 Talents Program of the Chinese Academy of Sciences.

\section{Appendix A. Supplementary material}

Supplementary data associated with this article can be found in the online version at http://dx.doi.org/10.1016/j.talanta.2013.12.019.

\section{References}

[1] Y. Lai, A. Ganguly, L. Chen, K. Chen, Biosens. Bioelectron. 26 (2010) 1688-1691. [2] S. Mitchell, G. Steventon, Drug Metab. Rev. 44 (2012) 129-147.

[3] V. Gazit, R. Ben-Abraham, R. Coleman, A. Weizman, Y. Katz, Amino Acids 26 (2004) 163-168.

[4] L. Chang, T. Wu, F. Chen, Microchim. Acta 177 (2012) 295-300.

[5] C. Chang, S. Lin, S. Wei, C. Yu, C. Lin, Anal. Bioanal. Chem. 402 (2012) 2827-2835.

[6] H. Hosseini, H. Ahmar, A. Dehghani, A. Bagheri, A. Tadjarodi, A. Fakhari, Biosens. Bioelectron. 42 (2013) 426-429.

[7] O. Nekrassova, N. Lawrence, R. Compton, Talanta 60 (2003) 1085-1095.

[8] P. Kubalczyk, E. Bald, Anal. Bioanal. Chem. 384 (2006) 1181-1185.

[9] Y. Wang, X. Kang, W. Ge, X. Sun, J. Peng, Chromatographia 65 (2007) 527-532.

[10] G. Chwatko, E. Bald, Talanta 52 (2000) 509-515.

[11] D. Negi, T. Chanu, Nanotechnology 19 (2008) 465503-465508.

[12] L. Xiong, Q. Zhao, H. Chen, Y. Wu, Z. Dong, Z. Zhou, F. Li, Inorg. Chem. 49 (2010) 6402-6408.

[13] W. Hao, A. McBride, S. McBride, J. Gao, Z. Wang, J. Mater. Chem. 21 (2011) 1040-1048.

[14] F. Tanaka, N. Mase, C. Barbas, Chem. Commun. 15 (2004) 1762-1763.

[15] H. Li, J. Fan, J. Wang, M. Tian, J. Du, S. Sun, P. Sun, X. Peng, Chem. Commun. 39 (2009) 5904-5906.

[16] J. Du, M. Hu, J. Fan, X. Peng, Chem. Soc. Rev. 41 (2012) 4511-4535.

[17] Y. Yang, Q. Zhao, W. Feng, F. Li, Chem. Rev. 113 (2013) 192-270.

[18] L. Chen, S. Xu, J. Li, Chem. Soc. Rev. 40 (2011) 2922-2942.

[19] X. Song, J. Li, S. Xu, R. Ying, J. Ma, C. Liao, D. Liu, J. Yu, L. Chen, Talanta 99 (2012) $75-82$.

[20] W. Cheong, S. Yang, F. Ali, J. Sep. Sci. 36 (2013) 609-628.

[21] J. Li, Y. Wen, L. Chen, Chin. J. Chromatogr. 31 (2013) 181-184.

[22] X. Shen, L. Zhu, N. Wang, L. Ye, H. Tang, Chem. Commun. 48 (2012) 788-798.

[23] J. Li, Z. Zhang, S. Xu, L. Chen, N. Zhou, H. Xiong, H. Peng, J. Mater. Chem. 21 (2011) 19267-19274.

[24] Y. Fuchs, O. Soppera, K. Haupt, Anal. Chim. Acta 717 (2012) 7-20.

[25] V. Syritski, J. Reut, A. Menaker, R. Gyurcsányi, A. Öpik, Electrochim. Acta 53 (2008) 2729-2736.

[26] M. Riskin, R. Tel-Vered, M. Frasconi, N. Yavo, I. Willner, Chem. Eur. J. 16 (2010) 7114-7120.

[27] Y. Zhang, P. Zhao, L. Yu, Sens. Actuators B-Chem. 181 (2013) 850-857.

[28] M. Siemann, L. Andersson, K. Mosbach, J. Agric. Food Chem. 44 (1996) $141-145$.

[29] X. Song, J. Li, J. Wang, L. Chen, Talanta 80 (2009) 694-702.

[30] W. Liao, F. Wu, Y. Wu, X. Wang, Microchim. Acta 162 (2008) 147-152.

[31] A. Nezamzadeh-Ejhieh, H. Hashemi, Talanta 88 (2012) 201-208.

[32] X. Liu, L. Luo, Y. Ding, Z. Kang, D. Ye, Bioelectrochemistry 86 (2012) 38-45.

[33] Y. Wang, J. Wang, F. Yang, X. Yang, Anal. Sci. 26 (2010) 545-549.

[34] X. Wei, L. Qi, J. Tan, R. Liu, F. Wang, Anal. Chim. Acta 671 (2010) 80-84.

[35] H. Alwael, D. Connolly, L. Barron, B. Paull, J. Chromatogr. A 1217 (2010) 3863-3870.

[36] X. Chen, T. Pradhan, F. Wang, J. Kim, J. Yoon, Chem. Rev. 112 (2012) 1910-1956. 